\title{
Insulin-Like Growth Factor-I Blocks Bcl-2 Interacting Mediator of Cell Death (Bim) Induction and Intrinsic Death Signaling in Cerebellar Granule Neurons
}

\author{
Daniel A. Linseman, Reid A. Phelps, Ron J. Bouchard, Shoshona S. Le, Tracey A. Laessig, Maria L. McClure, \\ and Kim A. Heidenreich \\ Department of Pharmacology, University of Colorado Health Sciences Center and the Denver Veterans Affairs Medical \\ Center, Denver, Colorado 80262
}

\begin{abstract}
Cerebellar granule neurons depend on insulin-like growth factor-I (IGF-I) for their survival. However, the mechanism underlying the neuroprotective effects of IGF-I is presently unclear. Here we show that IGF-I protects granule neurons by suppressing key elements of the intrinsic (mitochondrial) death pathway. IGF-I blocked activation of the executioner caspase-3 and the intrinsic initiator caspase-9 in primary cerebellar granule neurons deprived of serum and depolarizing potassium. IGF-I inhibited cytochrome $c$ release from mitochondria and prevented its redistribution to neuronal processes. The effects of IGF-I on cytochrome $c$ release were not mediated by blockade of the mitochondrial permeability transition pore, because IGF-I failed to inhibit mitochondrial swelling or depolarization. In contrast, IGF-I blocked induction of the $\mathrm{BH} 3-$ only $\mathrm{Bcl}-2$ family member,
\end{abstract}

Bim (Bcl-2 interacting mediator of cell death), a mediator of Bax-dependent cytochrome $c$ release. The suppression of Bim expression by IGF-I did not involve inhibition of the c-Jun transcription factor. Instead, IGF-I prevented activation of the forkhead family member, FKHRL1, another transcriptional regulator of Bim. Finally, adenoviral-mediated expression of dominant-negative AKT activated FKHRL1 and induced expression of Bim. These data suggest that IGF-I signaling via AKT promotes survival of cerebellar granule neurons by blocking the FKHRL1-dependent transcription of Bim, a principal effector of the intrinsic death-signaling cascade.

Key words: insulin-like growth factor; cerebellar granule neuron; apoptosis; mitochondria; Bim; forkhead transcription factor
Insulin-like growth factor-I (IGF-I) has significant neurotrophic and neuroprotective effects. IGF-I expression is regulated differentially in various brain regions and is associated temporally with critical stages of CNS development (Rotwein et al., 1988; Bach et al., 1991). Deficits in IGF-I are observed in Alzheimer's disease (Mustafa et al., 1999) and degenerative cerebellar ataxias (Torres-Aleman et al., 1996), and recombinant IGF-I slows disease progression in sporadic amyotrophic lateral sclerosis (Lai et al., 1997). IGF-I decreases neuronal apoptosis and enhances functional recovery in animal models of neurodegeneration including toxin exposure (Fernandez et al., 1998), transient ischemia (Liu et al., 2001), and neurotransplantation (Clarkson et al., 2001). Similarly, IGF-I rescues primary neurons from apoptosis induced by trophic factor withdrawal (Russell et al., 1998), excitotoxicity (Tagami et al., 1997), and oxidative stress (Heck et al., 1999). Thus IGF-I is essential for the survival of CNS neurons in vivo and in vitro.

Cerebellar granule neurons (CGNs) are critically dependent on IGF-I for their survival (Lin and Bulleit, 1997). In hereditary models of cerebellar Purkinje cell degeneration (pcd; lurcher), the primary death of Purkinje neurons induces the subsequent apo-

\footnotetext{
Received March 22, 2002; revised Aug. 5, 2002; accepted June 24, 2002.

This work was supported by a Department of Veterans Affairs Merit Award (K.A.H.), Department of Defense Grant DAMD17-99-1-9481 (K.A.H.), National Institutes of Health Grant NS38619-01A1 (K.A.H.), and a Department of Veterans Affairs Research Enhancement Award Program (K.A.H. and D.A.L.). We thank Mary Kay Meintzer for her help with preparation of this manuscript.

Correspondence should be addressed to Dr. Kim A. Heidenreich, Department of Pharmacology (C236), University of Colorado Health Sciences Center, 4200 East Ninth Avenue, Denver, CO 80262. E-mail: Kim.Heidenreich@UCHSC.edu. Copyright (C) 2002 Society for Neuroscience 0270-6474/02/229287-11\$15.00/0
}

ptosis of CGNs because of the loss of Purkinje-derived IGF-I (Bartlett et al., 1991; Zhang et al., 1997; Selimi et al., 2000a). Transgenic mice overexpressing IGF-I exhibit a remarkable doubling of CGN number (Ye et al., 1996) and show decreased expression of caspase-3 in cerebellum (Chrysis et al., 2001). These observations illustrate that IGF-I protects CGNs from apoptosis in vivo.

Similarly, IGF-I rescues primary CGNs from apoptosis induced by removal of depolarizing potassium and serum (trophic factor withdrawal), an established in vitro model of neuronal apoptosis (D’Mello et al., 1993; Galli et al., 1995; Miller et al., 1997a). CGN apoptosis involves activation of the intrinsic (mitochondrial) death pathway (Green, 1998). For example, trophic factordeprived CGNs demonstrate Bax-dependent cytochrome $c$ release from mitochondria (Desagher et al., 1999), and CGNs isolated from Bax knock-out mice are less sensitive to trophic factor withdrawal (Miller et al., 1997b). Moreover, the BH3-only proapoptotic Bcl-2 family member, Bim (Bcl-2 interacting mediator of cell death), is induced in CGNs undergoing apoptosis (Harris and Johnson, 2001; Putcha et al., 2001). BH3-only proteins facilitate intrinsic death signaling in a Bax-dependent manner (Desagher et al., 1999; Zong et al., 2001). Although it is recognized that IGF-I rescues CGNs via phosphatidylinositol 3 kinase (PI3K) and AKT (Dudek et al., 1997; Miller et al., 1997a), the effects of IGF-I on components of the intrinsic death pathway have not been examined.

Here we found that IGF-I suppresses induction of Bim, cytochrome $c$ release from mitochondria, and activation of the intrinsic initiator caspase- 9 and the executioner caspase- 3 in trophic factor-deprived CGNs. Although c-Jun N-terminal protein kinase 
$(\mathrm{JNK}) / \mathrm{c}-\mathrm{Jun}$ signaling has been implicated in the induction of Bim during neuronal apoptosis (Harris and Johnson, 2001; Whitfield et al., 2001), our data suggest that IGF-I suppresses Bim expression via a distinct mechanism involving inhibition of the forkhead transcription factor FKHRL1. These results indicate that the intrinsic death pathway is a principal target of IGF-I in neurons.

\section{MATERIALS AND METHODS}

Materials. Recombinant human IGF-I was provided by Margarita Quiroga (Chiron, Emeryville, CA). Polyclonal antibodies to Bim, Bcl- $\mathrm{X}_{\mathrm{L}}$, caspase-3, caspase-9, cytochrome $c$, and c-Jun were from Santa Cruz Biotechnology (Santa Cruz, CA). Polyclonal antibodies to phospho-c-Jun $\left(\mathrm{Ser}^{63}\right)$, phospho-AKT $\left(\mathrm{Ser}^{473}\right)$, and AKT were from Cell Signaling Technologies (Beverly, MA). Polyclonal antibodies to phosphoFKHRL1 (Ser ${ }^{253}$ ) and FKHRL1 were from Upstate Biotechnology (Lake Placid, NY). Cy3-conjugated secondary antibodies for immunocytochemistry were purchased from Jackson ImmunoResearch Laboratories (West Grove, PA). Horseradish peroxidase-linked secondary antibodies and reagents for enhanced chemiluminescence detection were obtained from Amersham Biosciences (Piscataway, NJ). JC1, tetramethylrhodamine ethyl ester (TMRE), and MitoTracker Green were from Molecular Probes (Eugene, OR). Wortmannin, Hoechst dye number 33258, and 4',6-diamidino-2-phenylindole (DAPI) were from Sigma (St. Louis, MO). Adenoviral cytokine response modifier A (CrmA) was obtained from Dr. James DeGregori [University of Colorado Health Sciences Center (UCHSC), Denver, CO]. Adenoviral CMV (negative control adenovirus) was from Dr. Jerry Schaack (UCHSC). Adenoviral kinase-dead K179M mutant (dominant-negative) AKT was obtained from Drs. Prem Sharma and Jerry Olefsky (University of California, San Diego, CA).

Cell culture. Rat CGNs were isolated from 7-d-old Sprague Dawley rat pups (15-19 gm) as described previously (Li et al., 2000). Briefly, neurons were plated on 35-mm-diameter plastic dishes coated with poly-L-lysine at a density of $2.0 \times 10^{6}$ cells $/ \mathrm{ml}$ in basal modified Eagle's medium containing $10 \%$ fetal bovine serum, $25 \mathrm{~mm} \mathrm{KCl,} 2 \mathrm{~mm}$ L-glutamine, and $100 \mathrm{U} / \mathrm{ml}$ penicillin $/ 100 \mu \mathrm{g} / \mathrm{ml}$ streptomycin (Invitrogen, Grand Island, NY). Cytosine arabinoside $(10 \mu \mathrm{M})$ was added to the culture medium 24 $\mathrm{hr}$ after plating to limit the growth of non-neuronal cells. With the use of this protocol the cultures were $\sim 95-99 \%$ pure for granule neurons. In general, experiments were performed after $7 \mathrm{~d}$ in culture.

Quantification of apoptosis. Apoptosis was induced by removing serum and decreasing the extracellular potassium concentration from 25 to 5 mM. After $24 \mathrm{hr}$ the CGNs were fixed with $4 \%$ paraformaldehyde, and the nuclei were stained with either Hoechst dye or DAPI. Cells were considered apoptotic if their nuclei either were condensed or were fragmented. In general, $\sim 500$ cells from at least two fields of a $35 \mathrm{~mm}$ well were counted. Data are presented as the percentage of cells in a given treatment group that were scored as apoptotic. Experiments were performed at least in triplicate.

Preparation of CGN cell extracts. After incubation for the indicated times and with the reagents specified in Results, the culture medium was aspirated; the cells were washed once with $2 \mathrm{ml}$ of ice-cold PBS, $\mathrm{pH} 7.4$, placed on ice, and scraped into lysis buffer $(200 \mu \mathrm{l} / 35 \mathrm{~mm}$ well) containing (in mM): 20 HEPES, pH 7.4, $50 \mathrm{NaCl}, 1$ EGTA, $5 \beta$-glycerophosphate, 30 sodium pyrophosphate, and 1 phenylmethylsulfonyl fluoride plus $1 \%$ Triton $\mathrm{X}-100,100 \mu \mathrm{M}$ sodium orthovanadate, $10 \mu \mathrm{g} / \mathrm{ml}$ leupeptin, and $10 \mu \mathrm{g} / \mathrm{ml}$ aprotinin. Cell debris was removed by centrifugation at $6000 \times g$ for $3 \mathrm{~min}$, and the protein concentration of the supernatant was determined by a commercially available protein assay kit (Pierce, Rockford, IL). Aliquots $(\sim 150 \mu \mathrm{g})$ of supernatant protein were diluted to a final concentration of $1 \times$ SDS-PAGE sample buffer, boiled for $5 \mathrm{~min}$, and electrophoresed through $10-15 \%$ polyacrylamide gels. Proteins were transferred to polyvinylidene difluoride (PVDF) membranes (Millipore, Bedford, MA) and processed for immunoblot analysis.

Immunoblot analysis. Nonspecific binding sites were blocked in PBS, $\mathrm{pH} 7.4$, containing $0.1 \%$ Tween 20 (PBS-T) and $1 \%$ BSA for $1 \mathrm{hr}$ at room temperature. Primary antibodies were diluted in blocking solution and incubated with the membranes for $1 \mathrm{hr}$. Excess primary antibody was removed by washing the membranes three times in PBS-T. Then the blots were incubated with the appropriate horseradish peroxidase-conjugated secondary antibody diluted in PBS-T for $1 \mathrm{hr}$ and subsequently were washed three times in PBS-T. Immunoreactive proteins were detected by enhanced chemiluminescence. In some experiments the membranes were reprobed after stripping in $0.1 \mathrm{M}$ Tris- $\mathrm{HCl}, \mathrm{pH} 8.0,2 \%$ SDS, and $100 \mathrm{~mm}$ $\beta$-mercaptoethanol for $30 \mathrm{~min}$ at $52^{\circ} \mathrm{C}$. The blots were rinsed twice in PBS-T and processed as above with a different primary antibody. Autoluminograms shown are representative of at least three independent experiments.

Immunocytochemistry. CGNs were cultured on polyethyleneiminecoated glass coverslips at a density of $\sim 2.5 \times 10^{5}$ cells per coverslip. After incubation as described in Results, the cells were fixed in $4 \%$ paraformaldehyde and were permeabilized and blocked in PBS, $\mathrm{pH} 7.4$, containing $0.2 \%$ Triton X-100 and 5\% BSA. Cells then were incubated for $\sim 16 \mathrm{hr}$ at $4^{\circ} \mathrm{C}$ with primary antibody diluted in PBS containing $0.2 \%$ Triton X-100 and 2\% BSA. The primary antibody was aspirated, and the cells were washed five times with PBS. Then the cells were incubated with a Cy3-conjugated secondary antibody and DAPI for $1 \mathrm{hr}$ at room temperature. CGNs were washed five more times with PBS, and coverslips were adhered to glass slides in mounting medium $(0.1 \%$ $p$-phenylenediamine in $75 \%$ glycerol in PBS). Fluorescent images were captured by using either $63 \times$ or $100 \times$ oil immersion objectives on a Zeiss Axioplan 2 microscope equipped with a Cooke Sensicam deep-cooled CCD camera and a Slidebook software analysis program for digital deconvolution (Intelligent Imaging Innovations, Denver, CO).

Measurement of mitochondrial swelling. CGNs were incubated as described in Results, and JC1 (final concentration, $2 \mu \mathrm{g} / \mathrm{ml}$ ) was added to the cultures $30 \mathrm{~min}$ before fixation to stain mitochondria. JC1 fluorescence was captured in paraformaldehyde-fixed cells by using a $\mathrm{Cy} 3$ filter under a $100 \times$ oil objective. Then the diameters of $\sim 150$ mitochondria per treatment condition were measured from digitally deconvolved images obtained from a total of 15-20 CGNs (randomly pooled from four independent experiments).

Assessment of mitochondrial membrane potential. CGNs grown on glass coverslips were incubated as described in Results, and TMRE (500 nM) was added directly to the cells $30 \mathrm{~min}$ before the end of the incubation period. After incubation the coverslips were inverted onto slides into a small volume of phenol red-free medium containing TMRE (500 nM). Living cells then were imaged with a $\mathrm{Cy} 3$ filter to detect TMRE fluorescence under a $100 \times$ oil objective. All images were acquired at equal exposure times for TMRE fluorescence to assess the relative mitochondrial membrane potentials.

Adenoviral infection. Recombinant adenoviruses were purified by cesium chloride gradient ultracentrif ugation. The viral titer (multiplicity of infection) was determined by measuring the absorbance at $260 \mathrm{~nm}$ (where 1.0 absorbance unit $=1 \times 10^{12}$ particles $/ \mathrm{ml}$ ), and infectious particles were verified by plaque assay. Adenoviral (Ad)-CMV, AdCrmA, or Ad-dominant-negative (DN)-AKT was added to CGN cultures on day 5 at a multiplicity of infection of 50 . At $48 \mathrm{hr}$ after infection either CGN apoptosis was induced by removal of serum and depolarizing potassium for $24 \mathrm{hr}$ (for Ad-CMV and Ad-CrmA) or cell lysates were prepared directly for Western blotting for phospho-FKHRL1 and Bim (for Ad-CMV and Ad-DN-AKT).

Data analysis. The results that are shown represent the means \pm SEM for the number $(n)$ of independent experiments that were performed. Statistical differences between the means of unpaired sets of data were evaluated by one-way ANOVA, followed by a post hoc Dunnett's test. A $p$ value of $<0.01$ was considered statistically significant.

\section{RESULTS}

\section{IGF-I suppresses CGN apoptosis and activation of caspase- 3 and caspase- 9}

Primary CGNs are dependent on depolarization-mediated calcium influx and serum-derived growth factors for their survival in vitro (D'Mello et al., 1993; Galli et al., 1995). The removal of serum and depolarizing potassium induced marked apoptosis of CGNs, characterized morphologically by chromatin condensation and fragmentation (Fig. 1A). Quantification of CGN apoptosis was performed by counting the number of cells with condensed and/or fragmented nuclei from several representative fields for each incubation condition. Basal CGN apoptosis was $\sim 10 \%$ and increased to $\sim 60 \%$ after $24 \mathrm{hr}$ of trophic factor withdrawal (Fig. $1 B)$. We used this cell system to investigate the mechanism of IGF-I neuroprotection. As described previously (D'Mello et al., 1993; Galli et al., 1995; Miller et al., 1997a), the addition of IGF-I 
A

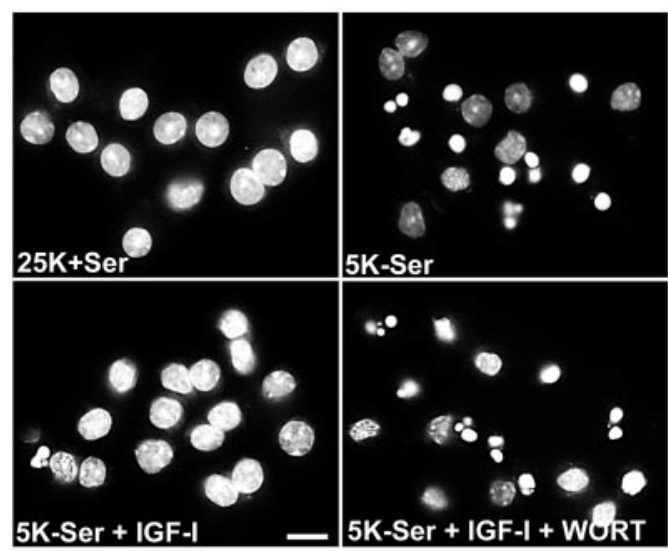

B

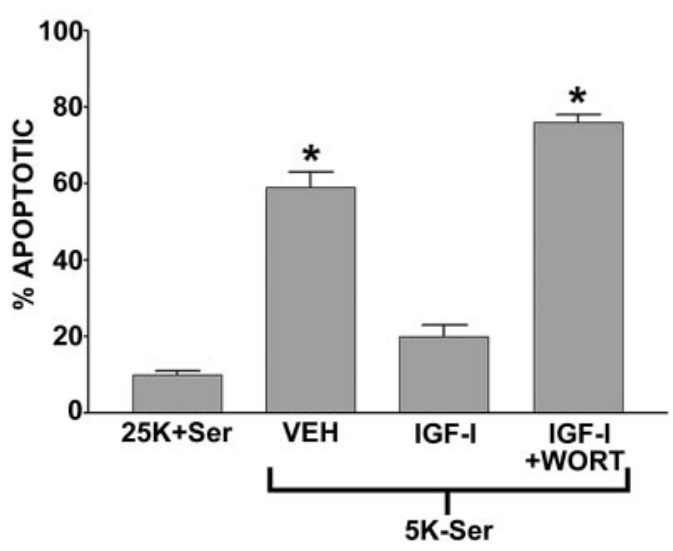

C

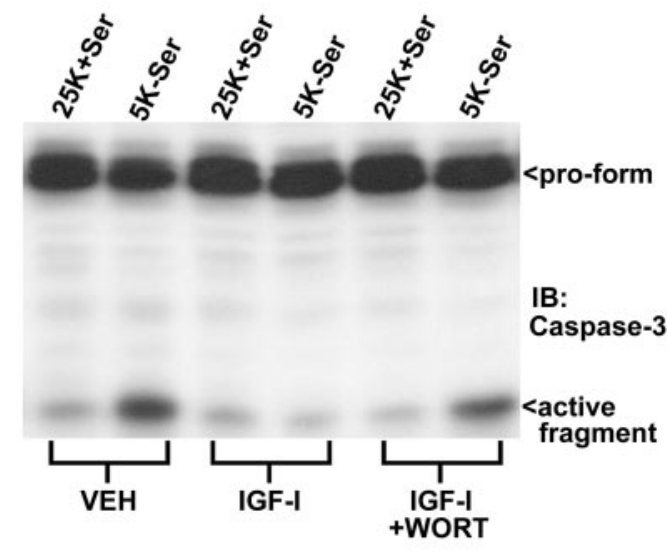

D

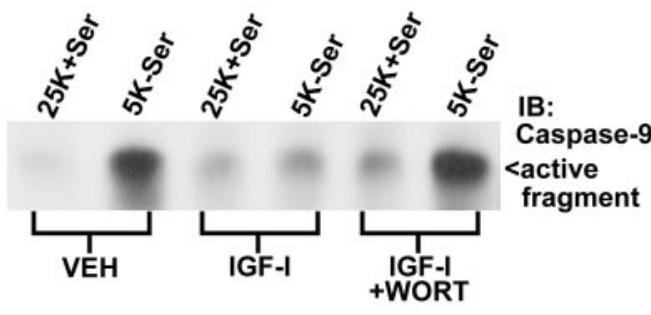

Figure 1. IGF-I inhibits apoptosis and activation of the executioner caspase-3 and the intrinsic initiator caspase-9 in CGNs subjected to trophic factor withdrawal. $A$, CGNs were incubated for $24 \mathrm{hr}$ in either control $(25 K+S e r)$ or apoptotic $(5 K-S e r)$ medium containing either PBS vehicle $(V E H)$ or IGF-I $(200 \mathrm{ng} / \mathrm{ml})$ in the absence or presence of wortmannin $(W O R T ; 100 \mathrm{nM})$. After incubation the CGNs were fixed, and the nuclei were stained with DAPI. Scale bar, $10 \mu \mathrm{m}$. $B$, The percentages of apoptotic CGNs observed under the conditions described in $A$ were quantified by counting $\sim 500$ CGNs per field in two fields per condition. to cerebellar cultures immediately after trophic factor withdrawal resulted in an $\sim 80 \%$ reduction in $\mathrm{CGN}$ apoptosis (Fig. $1 A, B$ ). The ability of IGF-I to rescue CGNs from apoptosis required the activation of PI3K, as demonstrated by the loss of protection observed in the presence of wortmannin (Fig. $1 A, B$ ). Activation of the executioner caspase- 3 has been implicated in the apoptotic death of CGNs (Eldadah et al., 2000). Consistent with this, we observed cleavage of caspase- 3 from the proform to an active fragment within $6 \mathrm{hr}$ of serum and potassium deprivation (Fig. $1 C)$. Like the results obtained for nuclear condensation and fragmentation, IGF-I inhibited caspase-3 activation in a PI3Kdependent manner (Fig. 1C). This latter result suggested that IGF-I blocks proapoptotic signaling events early in the apoptotic cascade, because caspase- 3 cleavage commonly is thought to signify commitment to apoptosis.

To identify potential targets of IGF-I action upstream of the executioner caspase- 3 in the apoptotic cascade, we focused on components of the intrinsic (mitochondrial) death pathway (Green, 1998). Recent data indicate that the intrinsic death pathway plays a significant role in CGN apoptosis evoked by trophic factor withdrawal (Miller et al., 1997b; Desagher et al., 1999). Immediately upstream of caspase-3 cleavage, activation of the initiator caspase- 9 is the most distal event in the intrinsic pathway (Kuida et al., 1998). Recently, caspase-9 activation was shown to be required for caspase-3 cleavage in CGNs deprived of serum and depolarizing potassium (Gerhardt et al., 2001). Consistent with the involvement of caspase-9 in CGN apoptosis, we found that infection of CGNs with adenoviral CrmA, an inhibitor of Group III caspases (including caspase-9), but not Group II caspases (such as caspase-3) (Garcia-Calvo et al., 1998), significantly decreased apoptosis from $72 \pm 8 \%(n=3)$ to $29 \pm 3 \%$ $(n=3 ; p<0.01)$. In contrast, a negative control adenovirus (Ad-CMV) had no effect on CGN apoptosis $(70 \pm 8 \% ; n=3)$. After acute serum and potassium deprivation, we observed marked cleavage of caspase-9 consistent with its activation (Fig. $1 D)$. As was observed for caspase-3 cleavage, activation of caspase-9 was inhibited significantly by IGF-I in a PI3Kdependent manner (Fig. 1D), demonstrating that IGF-I suppresses a key component of the intrinsic death pathway in CGNs.

\section{IGF-I inhibits release of cytochrome $c$ from mitochondria and its redistribution to neuronal processes}

Caspase- 9 is activated after its association with Apaf- 1 and cytochrome $c$, which assemble into a large oligomeric complex known as the apoptosome (Zou et al., 1999). Formation of the apoptosome occurs after release of the mitochondrial protein, cytochrome $c$, into the cytoplasm. In CGNs maintained in the pres-

\footnotetext{
Values represent the means \pm SEM for three independent experiments, each performed in triplicate. *Significantly different from the $25 \mathrm{~K}+\mathrm{Ser}$ control $(p<0.01)$. C, CGNs were incubated as described in $A$, but for only $6 \mathrm{hr}$. Detergent-soluble cell lysates were subjected to SDS-PAGE on $15 \%$ polyacrylamide gels, and the proteins were transferred to PVDF membranes. Activation of caspase- 3 was assessed by immunoblotting (IB) with a polyclonal antibody that recognizes both the proform $(\sim 32 \mathrm{kDa})$ and the cleaved form $(\sim 19 \mathrm{kDa}$ active fragment) of the enzyme. The blot shown is representative of results obtained in three separate experiments. $D$, CGNs were incubated exactly as described in $C$, and the cell lysates were electrophoresed as described in $C$. Activation of caspase-9 was assessed by Western blotting with a polyclonal antibody that specifically recognizes the cleaved form (active fragment) of the caspase. The blot shown is representative of three independent experiments.
} 

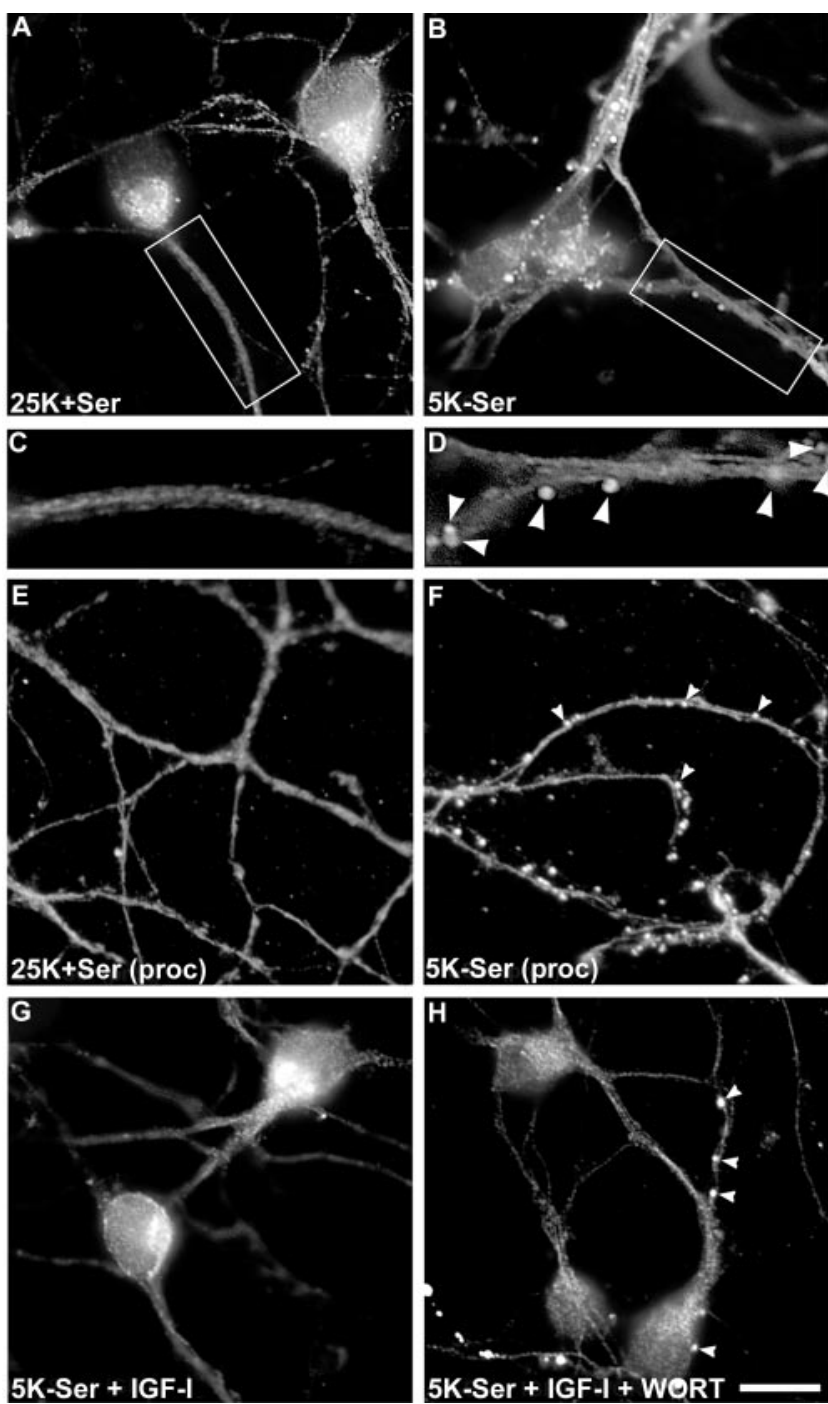

Figure 2. IGF-I blocks cytochrome $c$ release from mitochondria and prevents its redistribution to focal complexes localized in neuronal processes. CGNs were incubated for $4 \mathrm{hr}$ in control $(25 \mathrm{~K}+\mathrm{Ser})$ or apoptotic (5K-Ser) medium containing either PBS vehicle or IGF-I $(200 \mathrm{ng} / \mathrm{ml})$ in the absence or presence of wortmannin (WORT; $100 \mathrm{nM}$ ). After incubation the CGNs were fixed in $4 \%$ paraformaldehyde, permeabilized with $0.2 \%$ Triton X-100, and blocked with 5\% BSA. Cytochrome $c$ was localized by incubating the cells with a polyclonal antibody to cytochrome $c$ and a Cy3-conjugated secondary antibody. Digitally deconvolved images were captured by using a $63 \times$ oil objective. The images shown are representative of results obtained in three separate experiments. Scale bar, $10 \mu \mathrm{m}$. $A$, CGNs incubated in control medium demonstrated intense cytochrome $c$ staining in the perinuclear region consistent with localization to mitochondria. Very diffuse staining was observed in neuronal processes. $B$, CGNs incubated in apoptotic medium for $4 \mathrm{hr}$ showed a marked redistribution of cytochrome $c$. Note the overall diffuse staining throughout the cytoplasm accompanied by the formation of distinct, brightly fluorescent focal complexes on the cell bodies and processes. $C$, The area demarcated by the box in $A$ is enlarged to show the diffuse cytochrome $c$ staining in a control neuronal process. $D$, The area demarcated by the box in $B$ is enlarged to show the intense cytochrome $c$ staining localized to discrete focal complexes (indicated by the arrowheads) in neuronal processes of CGNs deprived of serum and depolarizing potassium. E, A region containing multiple processes ( proc) from control CGNs is shown. Note the overall diffuse cytochrome $c$ staining. $F$, A region containing multiple processes from CGNs incubated in apoptotic medium for $4 \mathrm{hr}$ is shown. Note the presence of many distinct focal areas of intense cytochrome $c$ staining (indicated by the arrowheads). $G$, CGNs incubated in apoptotic medium containing exogenous IGF-I displayed ence of serum and depolarizing potassium, cytochrome $c$ was localized predominantly in mitochondria (Fig. $2 A$ ), with only diffuse staining observed in neuronal processes (Fig. 2C,E). Removal of serum and depolarizing potassium for $4 \mathrm{hr}$ resulted in a rapid redistribution of cytochrome $c$ from mitochondria to a diffuse staining throughout the cytoplasm. This redistribution was accompanied by the formation of many pronounced punctate areas of cytochrome $c$ staining (Fig. 2B). The latter were observed primarily, although not exclusively, in distinct focal complexes localized to neuronal processes (Fig. 2D, F). In contrast to cytochrome $c$ staining, no detectable redistribution of the mitochondrial marker MitoTracker Green was observed in neuronal processes under apoptotic conditions, indicating that the punctate areas of cytochrome $c$ staining were not associated with intact mitochondria (data not shown). Inclusion of IGF-I during trophic factor withdrawal prevented the release and redistribution of cytochrome $c$ from mitochondria (Fig. 2G). However, the addition of wortmannin in combination with IGF-I restored the release of cytochrome $c$ from mitochondria and its redistribution to focal complexes in neuronal processes (Fig. $2 \mathrm{H}$ ), indicating that the effects of IGF-I on cytochrome $c$ release were PI3Kdependent. Thus, IGF-I inhibits the release of cytochrome $c$ from mitochondria and, in this manner, blocks the subsequent activation of the intrinsic initiator caspase-9.

\section{Mitochondrial swelling and mitochondrial membrane depolarization are not prevented by IGF-I}

There are two potential mechanisms underlying cytochrome $c$ release from mitochondria that have received considerable attention. The first involves opening of the permeability transition pore (PTP). The PTP is a heteromeric protein complex that includes the voltage-dependent anion channel, the adenine nucleotide translocator, and cyclophilin D as well as several other proteins (for review, see Martinou and Green, 2001). The PTP is localized at contact sites between the inner and outer mitochondrial membranes. Some apoptotic stimuli are capable of opening the PTP, resulting in disruption of the mitochondrial membrane potential (depolarization), a decline in ATP production, and entry of solutes and water into the mitochondrial matrix. Ultimately, mitochondrial swelling and rupture of the outer mitochondrial membrane occur, allowing the leakage of proteins (e.g., cytochrome $c$ ) from the intermembrane space into the cytoplasm. We used the mitochondrial dye JC1 to visualize mitochondria in CGNs undergoing apoptosis. Although the absolute amount of $\mathrm{JC} 1$ accumulated in mitochondria varies with membrane potential, JC1 is extremely photostable and labels all mitochondria to some extent (White and Reynolds, 1996). After incubation with JC1 the CGNs were fixed, and the diameters of labeled mitochondria were measured after digital deconvolution imaging. As shown in Figure 3, serum and potassium deprivation for $4 \mathrm{hr}$ resulted in dramatic swelling of mitochondria in CGNs (Fig. 3A, top panels). This effect was reversible if serum and depolarizing potassium were restored within the first $2 \mathrm{hr}$ after trophic factor withdrawal (Fig. $3 A$, bottom right panel). Inclusion of IGF-I during the apoptotic stimulus did not prevent the swelling of CGN

cytochrome $c$ localization to mitochondria similar to control CGNs (see $A$ for comparison). $H$, CGNs incubated in apoptotic medium containing both IGF-I and wortmannin showed cytochrome $c$ staining similar to CGNs incubated in apoptotic medium alone (see $B$ for comparison). Focal complexes of cytochrome $c$ staining are indicated by the arrowheads. 
A
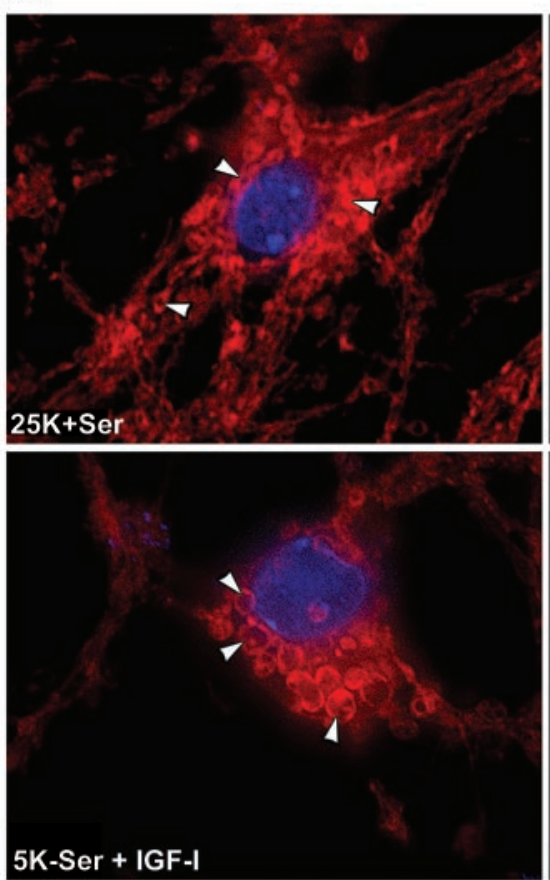

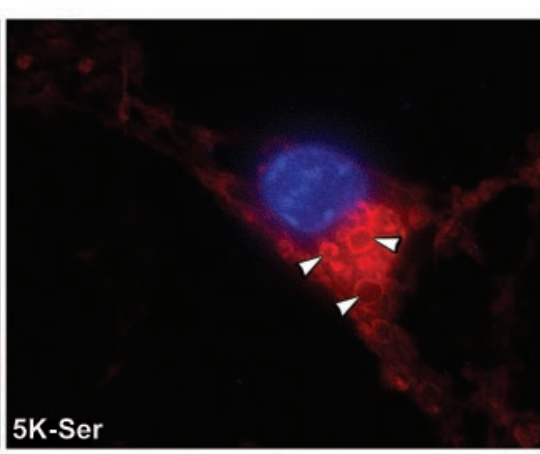

B

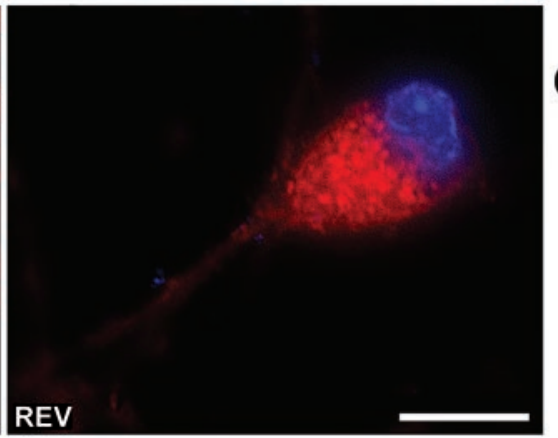

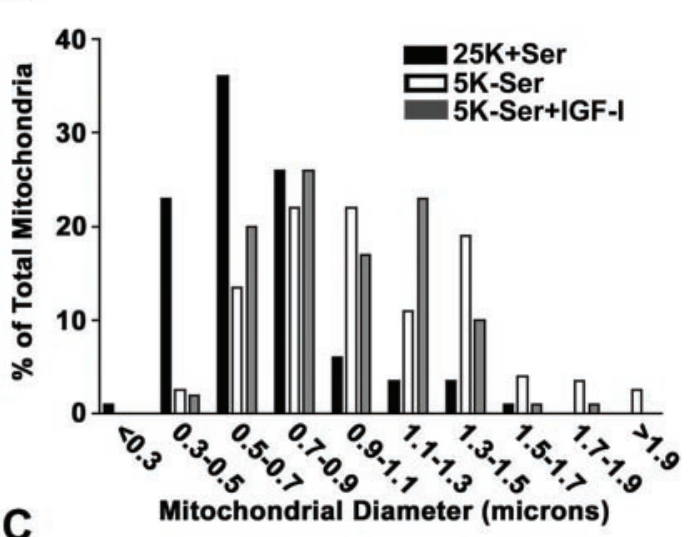

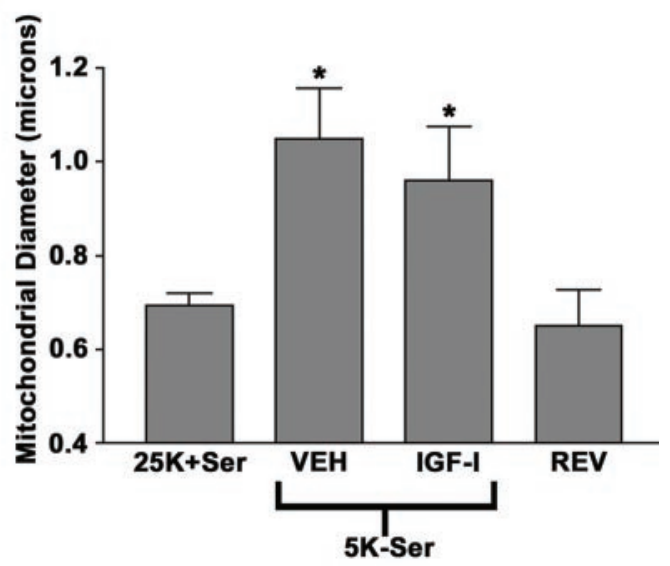

Figure 3. Mitochondrial swelling induced by trophic factor withdrawal is not inhibited by IGF-I. CGNs were incubated for 4 hr in either control $(25 K+$ Ser $)$ or apoptotic $(5 K-S e r)$ medium containing either PBS vehicle $(V E H)$ or IGF-I $(200 \mathrm{ng} / \mathrm{ml})$. To test for reversibility of mitochondrial swelling, we first incubated CGNs for $2 \mathrm{hr}$ in apoptotic medium and then returned them to control medium for an additional $2 \mathrm{hr}$ before staining (REV). In preliminary time course experiments a $2 \mathrm{hr}$ incubation in apoptotic medium was found to be sufficient to induce marked mitochondrial swelling (data not shown). At $30 \mathrm{~min}$ before fixation, JC1 (final concentration, $2 \mu \mathrm{g} / \mathrm{ml}$ ) and Hoechst dye were added to the cultures to stain mitochondria and nuclei, respectively. JC1 fluorescence was captured by using a Cy3 filter under a $100 \times$ oil objective. $A$, Representative images of CGNs incubated as described above and stained with Hoechst and JC1. Mitochondria are indicated by the arrowheads. Note the dramatic swelling of mitochondria in CGNs incubated in apoptotic medium in either the absence or presence of IGF-I. Mitochondrial swelling was completely reversible if control medium was replaced within $2 \mathrm{hr}(R E V)$. The images shown are representative of CGNs from four separate experiments. Scale bar, $10 \mu \mathrm{m}$. $B$, Distribution of mitochondrial diameters from CGNs observed under the conditions described in $A$. The diameters of $\sim 150$ mitochondria per treatment condition were measured from digitally deconvolved images obtained from a total of 15-20 CGNs (randomly pooled from 4 independent experiments). The mitochondrial diameters were categorized into the indicated size groups and graphed as a percentage of the total mitochondria with a given diameter $C$, Quantification of the mean mitochondrial diameters for CGNs observed under the conditions described in $A$. Values represent the means \pm SEM mitochondrial diameters obtained from the mitochondria measured in $B$. *Significantly different from the $25 \mathrm{~K}+$ Ser control; $p<0.01$.

mitochondria (Fig. 3A, bottom left panel). The distribution of mitochondrial diameters under control, apoptotic, and apoptotic plus IGF-I conditions is shown in Figure $3 B$, and the mean diameters are shown in Figure $3 C$. Serum and potassium deprivation resulted in a significant $50 \%$ increase in the mean mitochondrial diameter in CGNs $(0.69 \pm 0.03 \mu \mathrm{m}$ in control vs $1.05 \pm$ $0.11 \mu \mathrm{m}$ in apoptotic; $p<0.01)$, an effect that was unaltered by IGF-I $(0.96 \pm 0.12 \mu \mathrm{m})$ (Fig. $3 C)$. Interestingly, inclusion of IGF-I in trophic factor-deprived CGNs failed to reverse the mitochondrial swelling even after $48 \mathrm{hr}$ of incubation, although apoptosis was still blocked at this time point. However, the readdition of depolarizing potassium for the latter $24 \mathrm{hr}$ of the incubation period did reverse the mitochondrial swelling, indicating that it is a depolarization-sensitive event that is unaffected by IGF-I (data not shown).

Next we analyzed the mitochondrial membrane potential by incubating living CGNs with the membrane potential-sensitive dye TMRE. TMRE is accumulated actively in mitochondria possessing an intact membrane potential but is excluded or lost from mitochondria that are depolarized (Krohn et al., 1999). CGNs maintained in the presence of serum and depolarizing potassium displayed mitochondria that were stained brightly with TMRE, indicative of an intact membrane potential (Fig. 4, top left panel). Because these experiments were conducted in living CGNs, we showed that the mitochondrial membrane potential of control CGNs was maintained throughout the duration required to capture all of the images described below (Fig. 4, bottom right panel). Serum and potassium deprivation for $4 \mathrm{hr}$ resulted in a marked loss of mitochondrial TMRE staining, consistent with mitochondrial membrane depolarization (Fig. 4, top right panel). As was observed for mitochondrial swelling, the addition of IGF-I to trophic factor-deprived CGNs did not prevent the loss of mitochondrial membrane potential (Fig. 4, bottom left panel). The above results demonstrate that IGF-I does not block cytochrome 

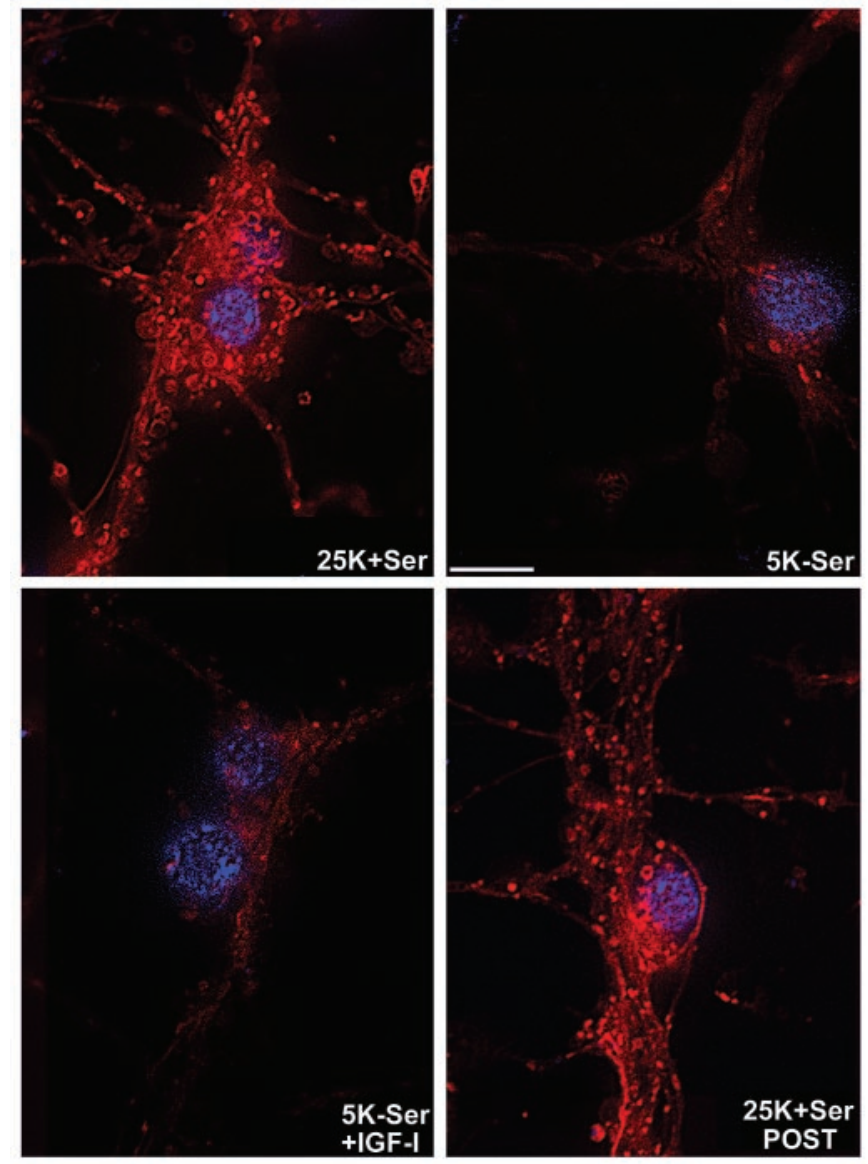

Figure 4. Mitochondrial membrane depolarization elicited by trophic factor withdrawal is not prevented by IGF-I. CGNs grown on glass coverslips were incubated for $4 \mathrm{hr}$ in either control $(25 \mathrm{~K}+\mathrm{Ser})$ or apoptotic $(5 K-S e r)$ medium alone or containing IGF-I $(200 \mathrm{ng} / \mathrm{ml})$. At $30 \mathrm{~min}$ before the end of the incubation period TMRE and Hoechst were added directly to the cells. After incubation the coverslips were inverted onto slides into a small volume of phenol red-free medium lacking serum or depolarizing potassium but containing TMRE (500 nM). Living cells then were imaged under a $100 \times$ oil objective. Nuclear staining with Hoechst is shown in blue; TMRE is shown in red. Scale bar, $10 \mu \mathrm{m}$. CGNs maintained in control medium during the $4 \mathrm{hr}$ incubation period displayed many mitochondria that were stained intensely with TMRE, indicative of an intact membrane potential (top left panel). In contrast, CGNs incubated in apoptotic medium in either the absence (top right panel) or presence (bottom left panel) of IGF-I expressed very little detectable mitochondrial TMRE staining, characteristic of a loss of mitochondrial membrane potential or depolarization. Approximately 5-10 min was required to capture the images described above. Therefore, at the end of the capture duration a final image was acquired of another control CGN to show that the mitochondrial membrane potential was not compromised during the time required to capture the images $(25 K+\operatorname{Ser} P O S T)$. All of the images shown were acquired at equal exposure times for TMRE fluorescence and are representative of results obtained from three independent experiments, each performed in duplicate.

$c$ release from CGN mitochondria by the inhibition of mitochondrial swelling or depolarization, suggesting that opening of the PTP does not play a significant role in cytochrome $c$ release during apoptosis of CGNs.

\section{IGF-I blocks induction of the BH3-only Bcl-2 family member Bim}

The second potential mechanism by which cytochrome $c$ release is regulated involves the formation of a Bax- or Bak-containing
A

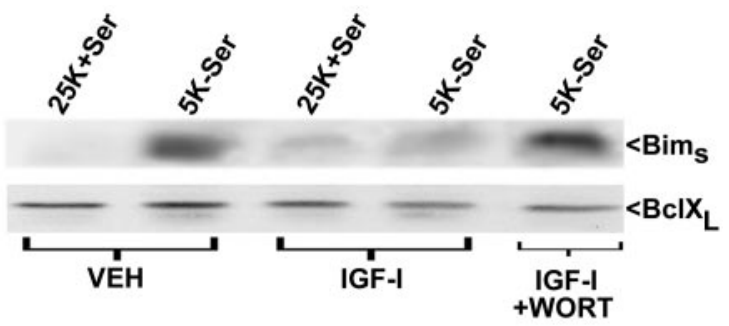

B

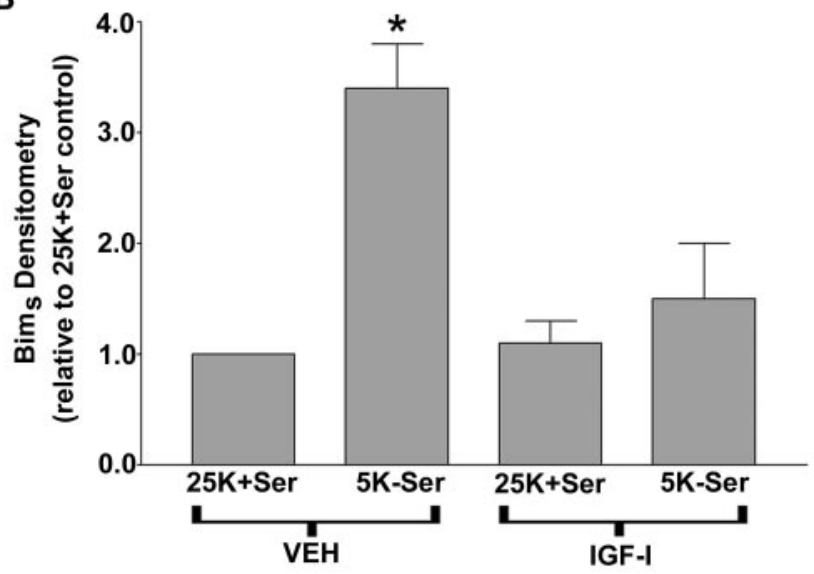

Figure 5. IGF-I inhibits induction of the BH3-only Bcl-2 family member Bim in a PI3K-dependent manner. $A$, CGNs were incubated for $6 \mathrm{hr}$ in either control $(25 \mathrm{~K}+\mathrm{Ser})$ or apoptotic $(5 \mathrm{~K}-\mathrm{Ser})$ medium containing either PBS vehicle $(V E H)$ or IGF-I $(200 \mathrm{ng} / \mathrm{ml}) \pm$ wortmannin $(W O R T ; 100$ nM). After incubation the cell lysates were subjected to SDS-PAGE on $15 \%$ polyacrylamide gels, and the proteins were transferred to PVDF membranes. Bim expression was assessed by immunoblotting with a polyclonal antibody to Bim that specifically recognized an $\sim 15 \mathrm{kDa}$ protein, consistent with the apparent molecular weight of Bim short $\left(\operatorname{Bim}_{s}\right)$. To affirm equal protein loading, we then stripped the blot and reprobed it for the anti-apoptotic Bcl-2 family member Bcl- $\mathrm{X}_{\mathrm{L}}$, which did not demonstrate any significant change in expression under the conditions of this experiment. The blots shown are representative of three separate experiments. $B$, Quantification of Bim $_{\mathrm{s}}$ expression observed under the conditions described in $A$ was performed by computer-assisted imaging densitometry. Values represent the means \pm SEM for three independent experiments and are expressed relative to the densitometry of Bim $_{\mathrm{s}}$ observed in control CGNs (set to 1.0). *Significantly different from the $25 \mathrm{~K}+$ Ser control $(p<0.01)$.

"pore" in the outer mitochondrial membrane that permits the passage of proteins (Korsmeyer et al., 2000). Bax and Bak are proapoptotic members of the Bcl-2 family that appear to serve a redundant function in making the mitochondrial membrane permeable to cytochrome $c$ (Wei et al., 2001). The BH3-only Bcl-2 family members, including Bad, Bid, Dp5/Hrk, and Bim, have been shown to promote the proapoptotic effects of Bax and Bak while concomitantly suppressing the prosurvival function of Bcl-2 (Desagher et al., 1999; Zong et al., 2001). Recently, Bim was shown to be upregulated after either nerve growth factor (NGF) withdrawal from primary sympathetic neurons or serum and potassium withdrawal from CGNs (Putcha et al., 2001; Whitfield et al., 2001). Moreover, overexpression of Bim or related BH3only family members promotes apoptosis of CGNs in a Baxdependent manner (Harris and Johnson, 2001). Immunoblotting for Bim after acute trophic factor withdrawal in CGNs demonstrated a marked increase in the expression of Bim short $\left(\mathrm{Bim}_{\mathrm{s}}\right.$, $\sim 15 \mathrm{kDa}$ ) (Fig. $5 A$ ), the most proapoptotic splice variant of this protein family (O'Connor et al., 1998). Quantification of the change in protein expression by densitometry revealed that serum 
and potassium deprivation for $6 \mathrm{hr}$ induced a significant $3.4 \pm$ 0.4 -fold increase $(n=3 ; p<0.01)$ in Bim $_{\mathrm{s}}$ (Fig. $\left.5 B\right)$. Inclusion of IGF-I completely blunted the induction of $\operatorname{Bim}_{\mathrm{s}}(1.5 \pm 0.5$-fold increase; $n=3$ ) in a PI3K-dependent manner (Fig. 5A,B). In contrast, the expression of the prosurvival Bcl-2 family member Bcl- $\mathrm{X}_{\mathrm{L}}$ was unaffected by either trophic factor withdrawal or IGF-I (Fig. 5A). These data indicate that the suppression of Bim is one mechanism by which IGF-I inhibits cytochrome $c$ release in CGNs deprived of serum and depolarizing potassium.

\section{IGF-I does not suppress Bim expression by inhibiting c-Jun}

Activation of the transcription factor c-Jun is required for apoptosis of primary sympathetic neurons subjected to NGF withdrawal (Eilers et al., 1998) and CGNs undergoing serum and potassium deprivation (Watson et al., 1998). The ability of a dominant-negative mutant of c-Jun to rescue sympathetic neurons from apoptosis recently has been attributed, in part, to its ability to block the induction of Bim (Whitfield et al., 2001). The transcriptional activity of c-Jun is stimulated after its phosphorylation on multiple serine residues (including $\operatorname{Ser}^{63}$ and Ser ${ }^{73}$ ) by upstream kinases of the JNK family (Minden et al., 1994). Chemical inhibitors of JNK have been shown to inhibit apoptosis of CGNs (Harada and Sugimoto, 1999; Coffey et al., 2002) and to attenuate Bim mRNA expression in CGNs subjected to trophic factor withdrawal (Harris and Johnson, 2001). In the present study we observed that incubation of trophic factor-deprived CGNs with the pyridinyl imidazole JNK/p38 inhibitor SB203580 (Harada and Sugimoto, 1999; Coffey et al., 2002) blunted the phosphorylation of c-Jun on $\operatorname{Ser}^{63}$ (Fig. 6A) and prevented the increased expression of c-Jun detected by immunoblotting (Fig. $6 B$ ). Moreover, SB203580 significantly attenuated the induction of $\mathrm{Bim}_{\mathrm{s}}$, consistent with a role for c-Jun in the regulation of Bim expression in CGNs (Fig. 6C,D). To determine whether IGF-I blocks Bim induction via an inhibition of c-Jun, we analyzed the effects of IGF-I on c-Jun phosphorylation and expression. The addition of IGF-I to CGNs deprived of serum and depolarizing potassium failed to attenuate either the phosphorylation of c-Jun on $\mathrm{Ser}^{63}$ (Fig. 6E) or the increased expression of c-Jun observed by Western blot (Fig. 6F). Thus IGF-I suppresses the induction of Bim in apoptotic CGNs via a mechanism that is independent of the transcription factor c-Jun.

\section{IGF-I prevents dephosphorylation and nuclear translocation of the forkhead transcription factor FKHRL1}

Recently, a member of the forkhead family of transcription factors, FKHRL1, was shown to regulate the induction of Bim in lymphocytes undergoing apoptosis in response to cytokine withdrawal (Dijkers et al., 2000). Furthermore, overexpression of a constitutively active mutant of FKHRL1 was sufficient to increase Bim expression in B-cells (Dijkers et al., 2000). The actions of forkhead family members are regulated by phosphorylation on serine and threonine residues. The prosurvival kinase AKT is the main effector of IGF-I that is activated downstream of PI3K, and FKHRL1 has been identified as a principal substrate of AKT in neuronal cells (Brunet et al., 1999; Zheng et al., 2000). Therefore, we first assessed the phosphorylation status of AKT and FKHRL1 in CGNs by immunoblotting with phospho-site-specific antibodies. CGNs cultured in the presence of serum and depolarizing potassium showed marked phosphorylation of AKT on $\mathrm{Ser}^{473}$, indicative of high AKT activity (Fig. $7 A$, first lane). In parallel, control CGNs also exhibited high phosphorylation of
FKHRL1 on Ser ${ }^{253}$, one of the sites targeted by AKT (Fig. 7B, first lane). Removal of serum and depolarizing potassium resulted in a pronounced dephosphorylation (inactivation) of AKT (Fig. $7 A$, second lane) and a corresponding loss of FKHRL1 phosphorylation (Fig. 7B, second lane). The addition of IGF-I to CGNs deprived of serum and depolarizing potassium maintained the phosphorylation of both AKT (Fig. 7A) and FKHRL1 (Fig. 7B), effects that were blocked by the PI3K inhibitor wortmannin.

Phosphorylation of FKHRL1 on $\mathrm{Thr}^{32}$ and $\mathrm{Ser}^{253}$ by AKT results in the translocation of FKHRL1 from the nucleus to the cytoplasm where it subsequently is sequestered by 14-3-3 proteins (Brunet et al., 1999). Thus IGF-I signaling via AKT has the potential to regulate negatively the transcriptional activity of FKHRL1 by excluding it from the nucleus. We next examined the cellular localization of FKHRL1 in CGNs. FKHRL1 was localized predominantly to the cytoplasm in CGNs maintained in the presence of trophic factors (Fig. 7C, top left panel). After acute trophic factor withdrawal FKHRL1 underwent a dramatic translocation to the nucleus (Fig. 7C, top right panel). The nuclear translocation of FKHRL1 was prevented completely by IGF-I (Fig. 7C, bottom left panel) in a PI3K-dependent manner (Fig. 7C, bottom right panel). Collectively, these results demonstrate that, under conditions in which IGF-I blocks Bim induction (Fig. 5), it concurrently sustains high AKT activity, robust FKHRL1 phosphorylation, and the exclusion of FKHRL1 from the nucleus. These findings are consistent with a role for FKHRL1 in the regulation of Bim expression in CGNs. Moreover, these data suggest a novel mechanism by which IGF-I suppresses Bim induction in trophic factor-deprived CGNs by blocking the actions of FKHRL1.

\section{Adenoviral-mediated expression of dominant-negative AKT results in dephosphorylation of FKHRL1 and induction of Bim}

To assess more directly the role of AKT in the regulation of FKHRL1 activity and Bim expression, we infected CGNs with an adenovirus expressing a kinase-dead dominant-negative mutant of AKT (Ad-DN-AKT). As described above, CGNs cultured in the presence of serum and depolarizing potassium showed marked phosphorylation of AKT on $\mathrm{Ser}^{473}$, indicative of high endogenous AKT activity (Fig. 7A, first lane). Under these conditions the adenoviral-mediated expression of DN-AKT resulted in a marked dephosphorylation of FKHRL1 on the AKT target site $\operatorname{Ser}^{253}$ (Fig. 8A) when compared with CGNs infected with a negative control adenovirus (Ad-CMV). Moreover, the dephosphorylation of FKHRL1 induced by DN-AKT was associated with a coordinated increase in the expression of Bim $_{\mathrm{s}}($ Fig. 8B). These data further support a mechanism by which IGF-I/AKT signaling blocks Bim induction at the level of the FKHRL1 transcription factor.

\section{DISCUSSION}

IGF-I promotes the survival of CGNs both in vitro and in vivo (Ye et al., 1996; Lin and Bulleit, 1997). In the current study we have investigated the neuroprotective mechanism of IGF-I in CGNs by systematically analyzing its effects on components of the intrinsic death-signaling cascade. First we found that IGF-I suppressed activation of the executioner caspase-3 in CGNs subjected to trophic factor withdrawal. This effect was blocked by the PI3K inhibitor wortmannin, consistent with a role for PI3K/AKT signaling in the IGF-I-mediated survival of CGNs. Previously, ribozyme-mediated downregulation of caspase-3 was shown to 
A

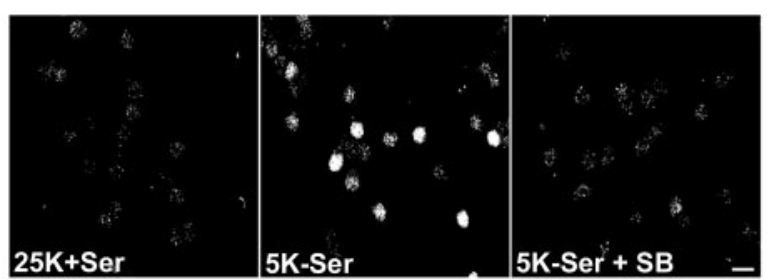

B
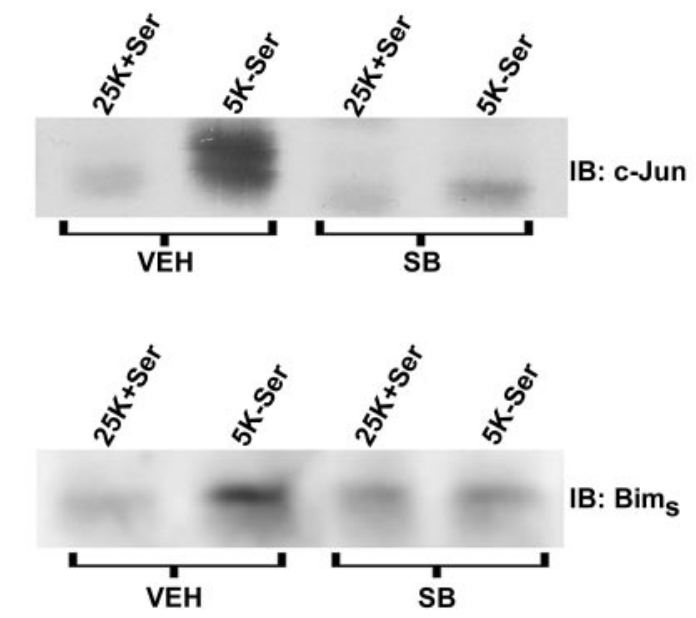

D

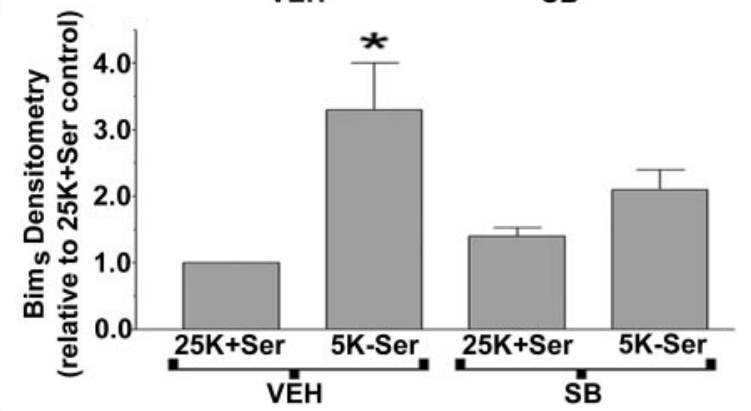

E
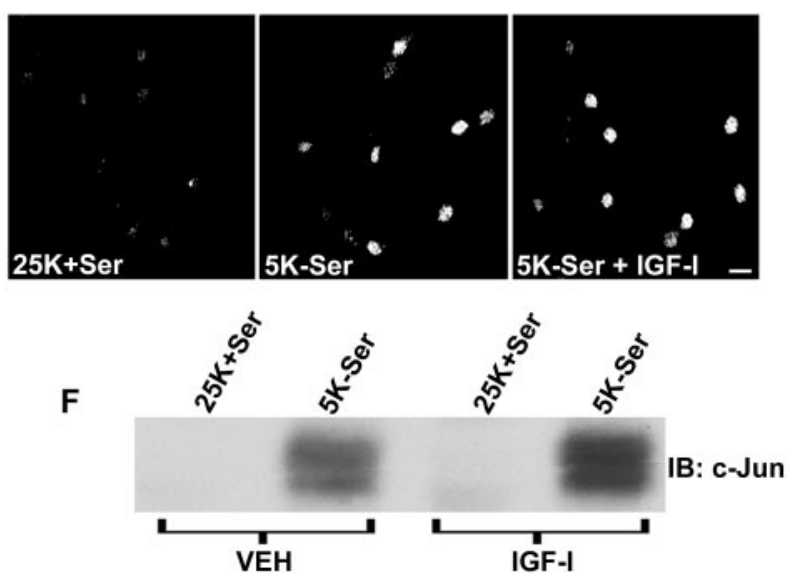

Figure 6. Inhibition of c-Jun signaling attenuates the induction of Bim. IGF-I does not block c-Jun activation in trophic factor-deprived CGNs. $A$, CGNs were incubated for $4 \mathrm{hr}$ in either control $(25 K+$ Ser $)$ or apoptotic $(5 K$-Ser $)$ medium in the absence or presence of the JNK/p38 inhibitor SB203580 $(S B ; 20 \mu \mathrm{M})$. After incubation the cells were fixed, and c-Jun phosphorylated on $\mathrm{Ser}^{63}$ was detected exclusively in the nuclear compartment by using a phospho-specific polyclonal antibody. The images shown are representative of results obtained in three independent experiments. Scale bar, $10 \mu \mathrm{m}$. B, CGNs were incubated for $4 \mathrm{hr}$ in either control $(25 \mathrm{~K}+\mathrm{Ser})$ or apoptotic $(5 \mathrm{~K}$-Ser $)$ medium in the presence of either DMSO vehicle $(V E H ; 0.2 \%)$ or SB $(20 \mu \mathrm{M})$, and cell lysates were subjected to SDS-PAGE on $10 \%$ polyacrylamide gels. Proteins were transferred to inhibit CGN apoptosis (Eldadah et al., 2000), supporting involvement of this executioner caspase in the mechanism of cell death. Comparable with our results, IGF-I has been shown to attenuate caspase-3 activation in other models of neuronal apoptosis via a PI3K/AKT-dependent mechanism (Van Golen and Feldman, 2000; Barber et al., 2001). We also found that IGF-I blocked activation of the intrinsic initiator caspase-9 in a PI3K-dependent manner. Recently, selective peptide inhibitors of caspase-9 were shown to prevent caspase-3 activation in CGNs, demonstrating that caspase-9 activation occurs upstream of the executioner during CGN apoptosis (Gerhardt et al., 2001). Moreover, we found that adenoviral CrmA, an inhibitor of caspase-9, protected CGNs from apoptosis. Similar to our data, IGF-I has been shown to prevent caspase- 9 activation in rat retinal ganglion cells after optic nerve transection (Kermer et al., 2000). Collectively, these data suggest a common mechanism by which IGF-I blocks activation of the executioner caspase- 3 in neurons via inhibition of the upstream intrinsic initiator caspase-9.

Because caspase- 9 is activated after its recruitment into the apoptosome, we analyzed the effects of IGF-I on cytochrome $c$ release from mitochondria. Acute trophic factor withdrawal from CGNs induced a rapid redistribution of cytochrome $c$ from mitochondria to focal complexes localized in neuronal processes. The precise nature of these complexes is currently under investigation, but it is possible that these cytochrome $c$-rich structures represent large aggregates of apoptosomes. This would allow for a localized activation of caspases in neuronal processes where many structural targets of these proteases exist (e.g., cytoskeletal proteins). We currently are attempting to colocalize Apaf-1 and caspase- 9 by immunocytochemical methods to these cytochrome $c$-containing complexes. Regardless of their exact content, IGF-I essentially abolished the formation of these complexes and maintained cytochrome $c$ in mitochondria. The effects of IGF-I on cytochrome $c$ redistribution were prevented by wortmannin, consistent with a previously recognized role for PI3K/AKT in the inhibition of cytochrome $c$ release (Kennedy et al., 1999). The above results suggest that IGF-I inhibits the activation of caspase- 9 by preventing the release of cytochrome $c$ and the subsequent formation of apoptosomes.

We next examined the role of the mitochondrial PTP in mediating the release of cytochrome $c$ during CGN apoptosis. In trophic factor-deprived CGNs marked mitochondrial swelling and depolarization were observed, indicative of PTP opening. Although IGF-I has been shown to prevent mitochondrial depolarization in neuroblastoma cells exposed to hyperosmotic conditions (Van Golen and Feldman, 2000), we did not observe any

$\leftarrow$

PVDF membranes and immunoblotted $(I B)$ with a polyclonal antibody to c-Jun. The blot shown is representative of three separate experiments. $C$, CGNs were incubated as described in $B$, but for $6 \mathrm{hr}$, and the cell lysates were electrophoresed on $15 \%$ polyacrylamide gels and probed for $\mathrm{Bim}_{\mathrm{s}}$. $D$, Densitometric analysis of $\mathrm{Bim}_{\mathrm{s}}$ from three independent experiments performed as described in $C$. The densitometry of $\mathrm{Bim}_{\mathrm{s}}$ detected in control $(25 K+S e r)$ CGNs was set at 1.0 , and all other values were calculated relative to the control. *Significantly different from the $25 \mathrm{~K}+\mathrm{Ser}$ control $(p<0.01)$. E, CGNs were incubated for $4 \mathrm{hr}$ in either control $(25 K+$ Ser $)$ or apoptotic $(5 K-S e r)$ medium in the presence of either PBS vehicle $(V E H)$ or IGF-I (200 ng/ml). After incubation the nuclear c-Jun phosphorylated on Ser $^{63}$ was detected by using a phospho-specific polyclonal antibody. The images shown are representative of three experiments. Scale bar, $10 \mu \mathrm{m}$. F, CGNs incubated as described in $E$ were lysed, and extracted proteins were immunoblotted for c-Jun. The blot shown is illustrative of results obtained from three separate experiments. 


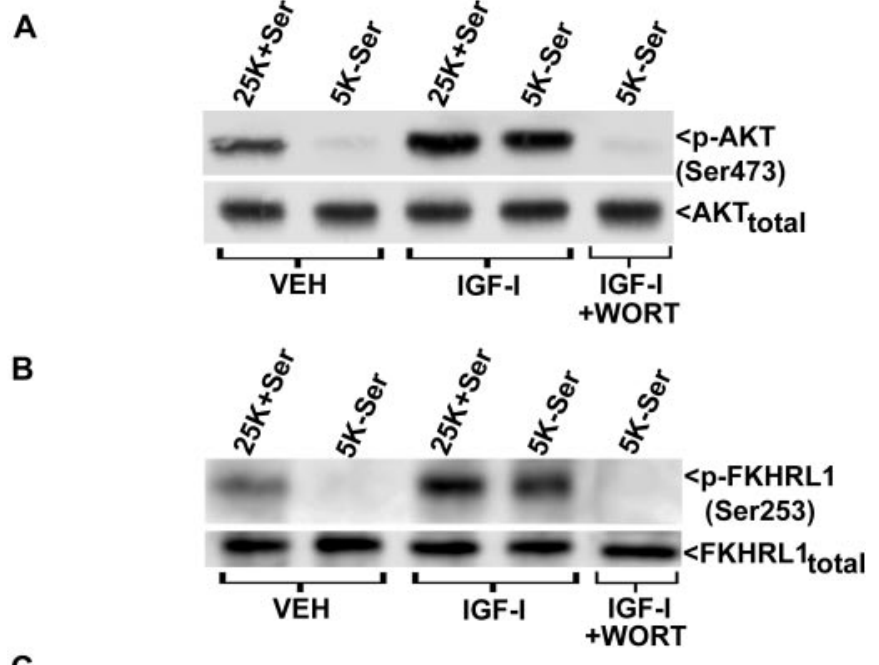

C
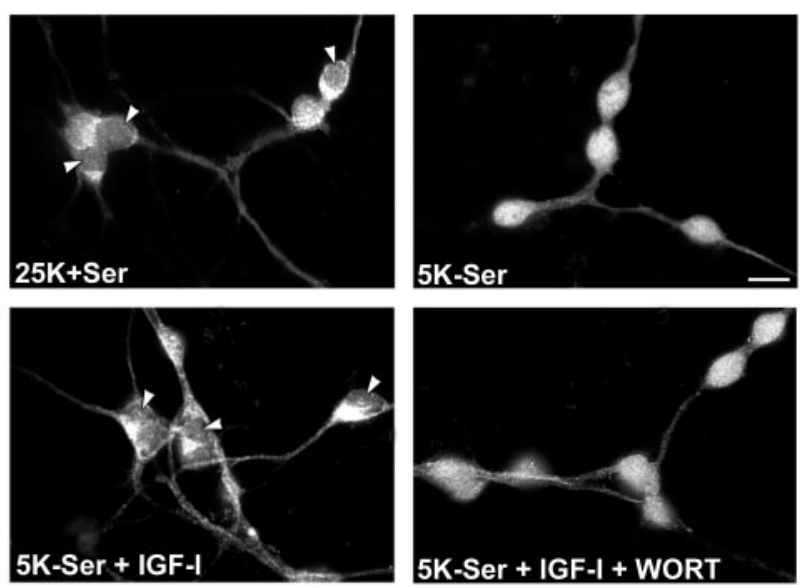

Figure 7. IGF-I sustains the phosphorylation of AKT and FKHRL1 and prevents the nuclear localization of FKHRL1 in CGNs deprived of trophic support. $A$, CGNs were incubated for $4 \mathrm{hr}$ in either control $(25 K+\mathrm{Ser})$ or apoptotic $(5 \mathrm{~K}$-Ser $)$ medium containing either PBS vehicle $(V E H)$ or IGF-I $(200 \mathrm{ng} / \mathrm{ml}) \pm$ wortmannin $(W O R T ; 100 \mathrm{nM})$. After incubation the cell lysates were subjected to SDS-PAGE on $10 \%$ polyacrylamide gels, and the proteins were transferred to PVDF membranes. Membranes were probed with a phospho-specific antibody that detects active AKT that was phosphorylated on $\operatorname{Ser}^{473}(p-A K T)$. Then the blots were stripped and reprobed for total AKT to demonstrate equal loading. The blots shown are typical of results obtained in three separate experiments. $B$, CGNs were treated exactly as described in $A$, and the cell lysates were immunoblotted for inactive FKHRL1 that was phosphorylated on $\operatorname{Ser}^{253}$ (p-FKHRL1) by using a phospho-specific antibody. Then the membranes were stripped and reprobed for total FKHRL1 to verify equal loading. The blots shown are illustrative of three independent experiments. $C$, CGNs incubated as described in $A$ were fixed and then incubated with a polyclonal antibody to FKHRL1, followed by a Cy3conjugated secondary antibody. Fluorescent digitally deconvolved images were acquired by using a $63 \times$ oil objective. The arrowheads indicate nuclei that are relatively devoid of FKHRL1 immunoreactivity. The images shown are representative of three separate experiments. Scale bar, $10 \mu \mathrm{m}$.

effect of IGF-I on either mitochondrial swelling or depolarization in CGNs. Although IGF-I clearly blocked apoptosis in trophic factor-deprived CGNs, the observation that it failed to prevent mitochondrial swelling indicates that the neurons still have damaged mitochondria in the presence of IGF-I. This finding suggests that a slower nonapoptotic death process was unmasked in CGNs by blocking the more rapid apoptotic death with IGF-I. The

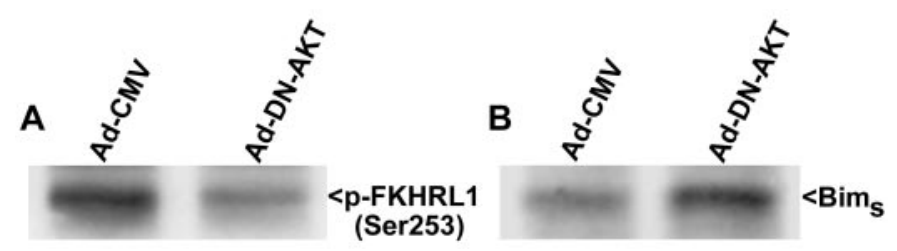

Figure 8. Adenoviral dominant-negative AKT induces dephosphorylation of FKHRL1 and increases Bim expression. On day 5 in culture the CGNs were infected with either a negative control adenovirus ( $A d-C M V)$ or adenovirus expressing kinase-dead (dominant-negative) AKT ( $A d$ $D N-A K T$ ), each at a multiplicity of infection of 50 . At $48 \mathrm{hr}$ after infection the cell lysates were subjected to SDS-PAGE on either $7.5 \%(p$ FKHRL1) or $15 \%\left(\operatorname{Bim}_{s}\right)$ polyacrylamide gels, and the proteins were transferred to PVDF membranes. $A$, The membrane was probed with a phospho-specific antibody to inactive FKHRL1 phosphorylated on Ser ${ }^{253}$ ( $p$-FKHRL1). B, The blot was probed with a polyclonal antibody that detects the short isoform of $\operatorname{Bim}\left(\operatorname{Bim}_{s}\right)$.

characterization of a nonapoptotic death pathway in CGNs will require further investigation. Nonetheless, because IGF-I blocked cytochrome $c$ release under conditions in which it failed to affect mitochondrial swelling or depolarization, we conclude that opening of the PTP is insufficient to promote cytochrome $c$ release in trophic factor-deprived CGNs. These results are in agreement with those previously reported for hippocampal neurons exposed to staurosporine in which cytochrome $c$ release and caspase-3 activation preceded mitochondrial depolarization (Krohn et al., 1999). Collectively, these results indicate that opening of the PTP may not be a principal mechanism for cytochrome $c$ release in neurons.

The proapoptotic Bcl-2 family member Bax has been implicated in cytochrome $c$ release and the apoptosis of CGNs in vitro and in vivo (Miller et al., 1997b; Desagher et al., 1999; Selimi et al., 2000b). The proapoptotic function of Bax is attenuated by anti-apoptotic members of the Bcl-2 family (Bcl-2, Bcl- $\left.\mathrm{X}_{\mathrm{L}}\right)$ that heterodimerize with Bax and sequester it away from mitochondria (Otter et al., 1998). Conversely, BH3-only Bcl-2 family members promote the proapoptotic effects of Bax by binding to $\mathrm{Bcl}-2$, thus freeing Bax to incorporate into the mitochondrial membrane (Zong et al., 2001). In addition, BH3-only proteins also have been shown to interact with Bax and induce a conformational change that facilitates its incorporation into mitochondria (Desagher et al., 1999). These findings illustrate that BH3-only proteins serve several key functions in the Bax-dependent release of cytochrome $c$ and initiation of the intrinsic death pathway.

The BH3-only protein Bim was shown recently to be induced both in sympathetic neurons subjected to NGF withdrawal and in trophic factor-deprived CGNs (Putcha et al., 2001; Whitfield et al., 2001). Sympathetic neuron apoptosis was attenuated by injection of Bim antisense oligonucleotides (Whitfield et al., 2001), and neurons from Bim knock-out mice were less sensitive to apoptosis than neurons from wild-type mice (Putcha et al., 2001). In addition, overexpression of Bim induced apoptosis in sympathetic neurons (Whitfield et al., 2001) and in CGNs in a Baxdependent manner (Harris and Johnson, 2001). Multiple isoforms of Bim have been identified that apparently arise by alternative splicing (O'Connor et al., 1998). In the works cited above (Putcha et al., 2001; Whitfield et al., 2001), Bim EL was induced during neuronal apoptosis, whereas we observed the induction of $\mathrm{Bim}_{\mathrm{s}}$ in CGNs subjected to trophic factor withdrawal. These isoform-specific differences may be a result of the specific antibodies used to detect Bim. The polyclonal antibody used in 
the current study detected primarily a single $\sim 15 \mathrm{kDa}$ protein in CGN lysates and in human embryonic kidney 293 cell lysates (data not shown), consistent with the apparent molecular weight of Bim $_{\mathrm{s}}$. We observed an approximately threefold induction of $\mathrm{Bim}_{\mathrm{s}}$ protein after acute trophic factor withdrawal that was prevented completely by inclusion of IGF-I in a PI3K-dependent manner. These results suggest that IGF-I suppresses the intrinsic death pathway upstream of Bim synthesis. Consistent with this conclusion, Harris and Johnson (2001) have shown recently that IGF-I was unable to rescue CGNs from apoptosis induced by overexpression of the $\mathrm{BH} 3$-only protein $\mathrm{Dp} 5 / \mathrm{Hrk}$. Thus our data are the first to identify the upregulation of Bim as a major target of IGF-I action in neurons undergoing apoptosis.

Bim expression is regulated by multiple transcription factors. In NGF-deprived sympathetic neurons dominant-negative c-Jun partially attenuated the induction of Bim mRNA and Bim $_{E L}$ protein, inhibited cytochrome $c$ release, and rescued sympathetic neurons from apoptosis (Whitfield et al., 2001). c-Jun also has been implicated in the apoptosis of CGNs (Watson et al., 1998), and an inhibitor of the JNK signaling pathway (CEP-1347) was shown recently to blunt partially the induction of Bim mRNA in CGNs subjected to trophic factor withdrawal (Harris and Johnson, 2001). In agreement with JNK/c-Jun involvement in the induction of $\mathrm{Bim}_{\mathrm{s}}$ in CGNs undergoing apoptosis, the p38/JNK inhibitor SB203580 significantly attenuated both the activation of c-Jun and the increase in Bim $_{\mathrm{s}}$ expression. However, IGF-I failed to inhibit c-Jun activation under conditions in which it significantly blocked the induction of Bim $_{\mathrm{s}}$. These results indicate that c-Jun plays a role in the regulation of Bim expression during CGN apoptosis, but IGF-I suppresses the induction of Bim via a mechanism that does not involve modulation of JNK/c-Jun signaling. This conclusion is in agreement with the work of Whitfield et al. (2001), who proposed that JNK/c-Jun signaling cooperates with a distinct JNK/c-Jun-independent pathway to stimulate the expression of Bim in sympathetic neurons deprived of NGF.

In this context the forkhead transcription factor FKHRL1 has been shown recently to regulate Bim expression in hematopoietic cells (Dijkers et al., 2000). Cytokine withdrawal from a pro-B cell line induced activation (dephosphorylation) of FKHRL1, induction of Bim, and apoptosis (Dijkers et al., 2000). Moreover, expression of a constitutively active mutant of FKHRL1, in which three putative AKT phosphorylation sites are mutated to alanine, induced Bim expression, cytochrome $c$ release, and apoptosis in hematopoietic cells (Dijkers et al., 2002). Given that FKHRL1 has been shown to be a substrate for AKT in neurons (Zheng et al., 2000), the AKT-mediated inactivation of FKHRL1 may be one mechanism by which IGF-I inhibits apoptosis. Indeed, overexpression of a constitutively active FKHRL1 triple phosphorylation site mutant is sufficient to induce the apoptosis of CGNs (Brunet et al., 1999). In the present study we showed that trophic factor withdrawal from CGNs led to an inactivation of AKT, a corresponding activation of FKHRL1, and translocation of FKHRL1 to the nucleus. All of these effects, along with the induction of Bim, were prevented by IGF-I in a PI3K-dependent manner. In addition, adenoviral expression of a dominant-negative mutant of AKT was sufficient to activate FKHRL1 and induce Bim expression in CGNs maintained in the presence of serum and depolarizing potassium. Taken together, our data suggest that IGF-I attenuates the induction of Bim in trophic factor-deprived CGNs via a PI3K/ AKT-mediated inactivation of the FKHRL1 transcription factor.

In summary, our results demonstrate that suppression of the intrinsic death signaling cascade is a principal mechanism under- lying the neuroprotective effects of IGF-I. IGF-I blocks Bim induction, cytochrome $c$ release, and activation of the intrinsic initiator caspase-9 and the executioner caspase-3 in CGNs deprived of trophic support. Moreover, IGF-I inhibits the actions of FKHRL1, a transcriptional regulator of Bim, suggesting a novel c-Jun-independent mechanism for the modulation of Bim in neurons.

\section{REFERENCES}

Bach MA, Shen-Orr Z, Lowe Jr WL, Roberts Jr CT, LeRoith D (1991) Insulin-like growth factor-I mRNA levels are developmentally regulated in specific regions of the rat brain. Brain Res Mol Brain Res 10:43-48.

Barber AJ, Nakamura M, Wolpert EB, Reiter CE, Seigel GM, Antonetti DA, Gardner TW (2001) Insulin rescues retinal neurons from apoptosis by a phosphatidylinositol 3-kinase/Akt-mediated mechanism that reduces the activation of caspase-3. J Biol Chem 276:32814-32821.

Bartlett WP, Li XS, Williams M, Benkovic S (1991) Localization of insulin-like growth factor-1 mRNA in murine central nervous system during postnatal development. Dev Biol 147:239-250.

Brunet A, Bonni A, Zigmond MJ, Lin MZ, Juo P, Hu LS, Anderson MJ, Arden KC, Blenis J, Greenberg ME (1999) Akt promotes cell survival by phosphorylating and inhibiting a Forkhead transcription factor. Cell 96:857-868.

Chrysis D, Calikoglu AS, Ye P, D'Ercole AJ (2001) Insulin-like growth factor-I overexpression attenuates cerebellar apoptosis by altering the expression of Bcl family proteins in a developmentally specific manner. J Neurosci 21:1481-1489.

Clarkson ED, Zawada WM, Bell KP, Esplen JE, Choi PK, Heidenreich KA, Freed CR (2001) IGF-I and bFGF improve dopamine neuron survival and behavioral outcome in parkinsonian rats receiving cultured human fetal tissue strands. Exp Neurol 168:183-191.

Coffey ET, Smiciene G, Hongisto V, Cao J, Brecht S, Herdegen T, Courtney MJ (2002) c-Jun N-terminal protein kinase (JNK) 2/3 is specifically activated by stress, mediating c-Jun activation, in the presence of constitutive JNK1 activity in cerebellar neurons. J Neurosci 22:4335-4345.

Desagher S, Osen-Sand A, Nichols A, Eskes R, Montessuit S, Lauper S, Maundrell K, Antonsson B, Martinou JC (1999) Bid-induced conformational change of Bax is responsible for mitochondrial cytochrome $c$ release during apoptosis. J Cell Biol 144:891-901.

Dijkers PF, Medema RH, Lammers JW, Koenderman L, Coffer PJ (2000) Expression of the pro-apoptotic Bcl-2 family member Bim is regulated by the forkhead transcription factor FKHR-L1. Curr Biol 10:1201-1204.

Dijkers PF, Birkenkamp KU, Lam EW, Thomas NS, Lammers JW, Koenderman L, Coffer PJ (2002) FKHR-L1 can act as a critical effector of cell death induced by cytokine withdrawal: protein kinase B-enhanced cell survival through maintenance of mitochondrial integrity. J Cell Biol 156:531-542.

D'Mello SR, Galli C, Ciotti T, Calissano P (1993) Induction of apoptosis in cerebellar granule neurons by low potassium: inhibition of death by insulin-like growth factor-I and cAMP. Proc Natl Acad Sci USA 90:10989-10993.

Dudek H, Datta SR, Franke TF, Birnbaum MJ, Yao R, Cooper GM, Segal RA, Kaplan DR, Greenberg ME (1997) Regulation of neuronal survival by the serine-threonine protein kinase Akt. Science 275:661-665.

Eilers A, Whitfield J, Babij C, Rubin LL, Ham J (1998) Role of the Jun kinase pathway in the regulation of c-Jun expression and apoptosis in sympathetic neurons. J Neurosci 18:1713-1724.

Eldadah BA, Ren RF, Faden AI (2000) Ribozyme-mediated inhibition of caspase-3 protects cerebellar granule cells from apoptosis induced by serum-potassium deprivation. J Neurosci 20:179-186.

Fernandez AM, de la Vega AG, Torres-Aleman I (1998) Insulin-like growth factor-I restores motor coordination in a rat model of cerebellar ataxia. Proc Natl Acad Sci USA 95:1253-1258.

Galli C, Meucci O, Scorziello A, Werge TM, Calissano P, Schettini G (1995) Apoptosis in cerebellar granule cells is blocked by high $\mathrm{KCl}$, forskolin, and IGF-1 through distinct mechanisms of action: the involvement of intracellular calcium and RNA synthesis. J Neurosci 15:1172-1179.

Garcia-Calvo M, Peterson EP, Leiting B, Ruel R, Nicholson DW, Thornberry NA (1998) Inhibition of human caspases by peptide-based and macromolecular inhibitors. J Biol Chem 273:32608-32613.

Gerhardt E, Kugler S, Leist M, Beier C, Berliocchi L, Volbracht C, Weller M, Bahr M, Nicotera P, Schulz JB (2001) Cascade of caspase activation in potassium-deprived cerebellar granule neurons: targets for treatment with peptide and protein inhibitors of apoptosis. Mol Cell Neurosci 17:717-731.

Green DR (1998) Apoptotic pathways: the roads to ruin. Cell 94:695-698. 
Harada J, Sugimoto M (1999) An inhibitor of p38 and JNK MAP kinases prevents activation of caspase and apoptosis of cultured cerebellar granule neurons. Jpn J Pharmacol 79:369-378.

Harris CA, Johnson Jr EM (2001) BH3-only Bcl-2 family members are coordinately regulated by the JNK pathway and require Bax to induce apoptosis in neurons. J Biol Chem 276:37754-37760.

Heck S, Lezoualc'h F, Engert S, Behl C (1999) Insulin-like growth factor-1-mediated neuroprotection against oxidative stress is associated with activation of nuclear factor $\kappa$ B. J Biol Chem 274:9828-9835.

Kennedy SG, Kandel ES, Cross TK, Hay N (1999) Akt/protein kinase B inhibits cell death by preventing the release of cytochrome $c$ from mitochondria. Mol Cell Biol 19:5800-5810.

Kermer P, Ankerhold R, Klocker N, Krajewski S, Reed JC, Bahr M (2000) Caspase-9: involvement in secondary death of axotomized rat retinal ganglion cells in vivo. Brain Res Mol Brain Res 85:144-150.

Korsmeyer SJ, Wei MC, Saito M, Weiler S, Oh KJ, Schlesinger PH (2000) Pro-apoptotic cascade activates BID, which oligomerizes BAK or BAX into pores that result in the release of cytochrome $c$. Cell Death Differ 7:1166-1173.

Krohn AJ, Wahlbrink T, Prehn JH (1999) Mitochondrial depolarization is not required for neuronal apoptosis. J Neurosci 19:7394-7404.

Kuida K, Haydar TF, Kuan CY, Gu Y, Taya C, Karasuyama H, Su MS, Rakic P, Flavell RA (1998) Reduced apoptosis and cytochrome $c$ mediated caspase activation in mice lacking caspase-9. Cell 94:325-337.

Lai EC, Felice KJ, Festoff BW, Gawel MJ, Gelinas DF, Kratz R, Murphy MF, Natter HM, Norris FH, Rudnicki SA (1997) Effect of recombinant human insulin-like growth factor-I on progression of ALS. A placebo-controlled study. The North America ALS/IGF-I study group. Neurology 49:1621-1630.

Li M, Wang X, Meintzer MK, Laessig T, Birnbaum MJ, Heidenreich KA (2000) Cyclic AMP promotes neuronal survival by phosphorylation of glycogen synthase kinase 3 $\beta$. Mol Cell Biol 20:9356-9363.

Lin X, Bulleit RF (1997) Insulin-like growth factor-I (IGF-I) is a critical trophic factor for developing cerebellar granule cells. Brain Res Dev Brain Res 99:234-242.

Liu XF, Fawcett JR. Thorne RG, Frey II WH (2001) Non-invasive intranasal insulin-like growth factor-I reduces infarct volume and improves neurologic function in rats following middle cerebral artery occlusion. Neurosci Lett 308:91-94.

Martinou JC, Green DR (2001) Breaking the mitochondrial barrier. Nat Rev Mol Cell Biol 2:63-67.

Miller TM, Tansey MG, Johnson Jr EM, Creedon DJ (1997a) Inhibition of phosphatidylinositol 3-kinase activity blocks depolarization- and insulin-like growth factor-I-mediated survival of cerebellar granule cells. J Biol Chem 272:9847-9853.

Miller TM, Moulder KL, Knudson CM, Creedon DJ, Deshmukh M, Korsmeyer SJ, Johnson Jr EM (1997b) Bax deletion further orders the cell death pathway in cerebellar granule cells and suggests a caspaseindependent pathway to cell death. J Cell Biol 139:205-217.

Minden A, Lin A, Smeal T, Derijard B, Cobb M, Davis R, Karin M (1994) c-Jun N-terminal phosphorylation correlates with activation of the JNK subgroup but not the ERK subgroup of mitogen-activated protein kinases. Mol Cell Biol 14:6683-6688.

Mustafa A, Lannfelt L, Lilius L, Islam A, Winblad B, Adem A (1999) Decreased plasma insulin-like growth factor-I level in familial Alzheimer's disease patients carrying the Swedish APP 670/671 mutation. Dement Geriatr Cogn Disord 10:446-451.

O'Connor L, Strasser A, O'Reilly LA, Hausmann G, Adams JM, Cory S, Huang DC (1998) Bim: a novel member of the Bcl-2 family that promotes apoptosis. EMBO J 17:384-395.

Otter I, Conus S, Ravn U, Rager M, Olivier R, Monney L, Fabbro D,
Borner C (1998) The binding properties and biological activities of Bcl-2 and Bax in cells exposed to apoptotic stimuli. J Biol Chem 273:6110-6120.

Putcha GV, Moulder KL, Golden JP, Bouillet P, Adams JA, Strasser A, Johnson Jr EM (2001) Induction of Bim, a proapoptotic BH3-only Bcl-2 family member, is critical for neuronal apoptosis. Neuron 29:615-628.

Rotwein P, Burgess SK, Milbrandt JD, Krause JE (1988) Differential expression of insulin-like growth factor genes in rat central nervous system. Proc Natl Acad Sci USA 85:265-269.

Russell JW, Windebank AJ, Schenone A, Feldman EL (1998) Insulinlike growth factor-I prevents apoptosis in neurons after nerve growth factor withdrawal. J Neurobiol 36:455-467.

Selimi F, Doughty M, Delhaye-Bouchaud N, Mariani J (2000a) Targetrelated and intrinsic neuronal death in lurcher mutant mice are both mediated by caspase-3 activation. J Neurosci 20:992-1000.

Selimi F, Vogel MW, Mariani J (2000b) Bax inactivation in lurcher mutants rescues cerebellar granule cells but not Purkinje cells or inferior olivary neurons. J Neurosci 20:5339-5345.

Tagami M, Yamagata K, Nara Y, Fujino H, Kubota A, Numano F, Yamori Y (1997) Insulin-like growth factors prevent apoptosis in cortical neurons isolated from stroke-prone spontaneously hypertensive rats. Lab Invest 76:603-612.

Torres-Aleman I, Barrios V, Lledo A, Berciano J (1996) The insulinlike growth factor-I system in cerebellar degeneration. Ann Neurol 39:335-342

Van Golen CM, Feldman EL (2000) Insulin-like growth factor-I is the key growth factor in serum that protects neuroblastoma cells from hyperosmotic-induced apoptosis. J Cell Physiol 182:24-32.

Watson A, Eilers A, Lallemand D, Kyriakis J, Rubin LL, Ham J (1998) Phosphorylation of c-Jun is necessary for apoptosis induced by survival signal withdrawal in cerebellar granule neurons. J Neurosci 18:751-762.

Wei MC, Zong W X, Cheng EH, Lindsten T, Panoutsakopoulou V, Ross AJ, Roth KA, MacGregor GR, Thompson CB, Korsmeyer SJ (2001) Proapoptotic BAX and BAK: a requisite gateway to mitochondrial dysfunction and death. Science 292:727-730.

White RJ, Reynolds IJ (1996) Mitochondrial depolarization in glutamatestimulated neurons: an early signal specific to excitotoxin exposure. J Neurosci 16:5688-5697.

Whitfield J, Neame SJ, Paquet L, Bernard O, Ham J (2001) Dominantnegative c-Jun promotes neuronal survival by reducing Bim expression and inhibiting mitochondrial cytochrome $c$ release. Neuron 29:629-643.

Ye P, Xing Y, Dai Z, D'Ercole AJ (1996) In vivo actions of insulin-like growth factor-I (IGF-I) on cerebellum development in transgenic mice: evidence that IGF-I increases proliferation of granule cell progenitors. Brain Res Dev Brain Res 95:44-54.

Zhang W, Ghetti B, Lee WH (1997) Decreased IGF-I gene expression during the apoptosis of Purkinje cells in $p c d$ mice. Brain Res Dev Brain Res 98:164-176.

Zheng WH, Kar S, Quirion R (2000) Insulin-like growth factor-1induced phosphorylation of the forkhead family transcription factor FKHRL1 is mediated by Akt kinase in PC12 cells. J Biol Chem 275:39152-39158.

Zong WX, Lindsten T, Ross AJ, MacGregor GR, Thompson CB (2001) BH3-only proteins that bind pro-survival Bcl-2 family members fail to induce apoptosis in the absence of Bax and Bak. Genes Dev 15:1481-1486.

Zou H, Li Y, Liu X, Wang X (1999) An APAF-1•cytochrome $c$ multimeric complex is a functional apoptosome that activates procaspase-9. J Biol Chem 274:11549-11556. 